\title{
Simulation model of robot supported standing-up in paraplegia
}

\author{
R. Kamnik, J. Kuželički \& T. Bajd \\ University of Ljubljana \\ Faculty of Electrical Engineering \\ Tržaška 25, 1000 Ljubljana, Slovenia
}

\begin{abstract}
For the standing-up rehabilitation, a robot assistive device is proposed to provide support for impaired individuals when rising from a sitting to a standing position. In this paper, a simulation model of the robot supported standing-up of paraplegic subject is presented. The simulation model describes the behaviour of a human body during rising when interacting with an active device. In the model, the voluntary activity of the upper body, the visco-elastic properties of paralyzed lower extremities and the subject/robot dynamic interaction are incorporated. The equations of motion are derived by the help of SD/FAST software package, while the model was implemented in the Matlab-Simulink simulation environment. The simulation results were evaluated with the measurement results of a real robot supported standing-up. The results proved the adequate and reliable performance of the simulation model.
\end{abstract}

\section{Introduction}

Rising from a chair is a common but demanding activity of daily living. Impaired persons and the elderly often have difficulty when rising to a standing position. There are many reasons that cause this difficulty, such as pain, muscle weakness, partial loss of motion control or physical deformity of the joint structures. Conse- 
quently, individuals experiencing rising difficulties have problems living independently, while their prolonged immobilization results in physiological problems. The regular standing-up and standing activity is supposed to ameliorate some of the problems.

To compensate for the lack of lifting forces, a handicapped person usually develops an adapted approach to standing-up. An additional aid is normally utilized in this activity. It has been shown that in the rising of healthy subjects utilization of the arm support substantially reduces the net moments in the lower extremity joints $[1,2,3]$. However, a person practicing a fully arm supported rising risks later complications of the upper extremity joints [4]. Additionally, various mechanical constructions can be employed to facilitate the rising. These mechanisms are typically based upon passive principles, exploiting spring or counterweight forces $[5,6,7,8]$. However, neither of the devices provides the feedback information about the rising process and the capability of motion trajectory programming. Hence, for the purposes of standing-up training and rehabilitation, a novel robot assistive device is proposed to provide support for impaired individuals when rising from a sitting to a standing position. The robot device is designed as a 3 DOF mechanism driven by an electrohydraulic servosystem supporting the rising subject under the buttocks. The actuators and the control system are designed to allow the device to operate in a position or in force control mode. The device, presented in Figure 1, is instrumented with a sensory system providing information about the standing-up parameters.

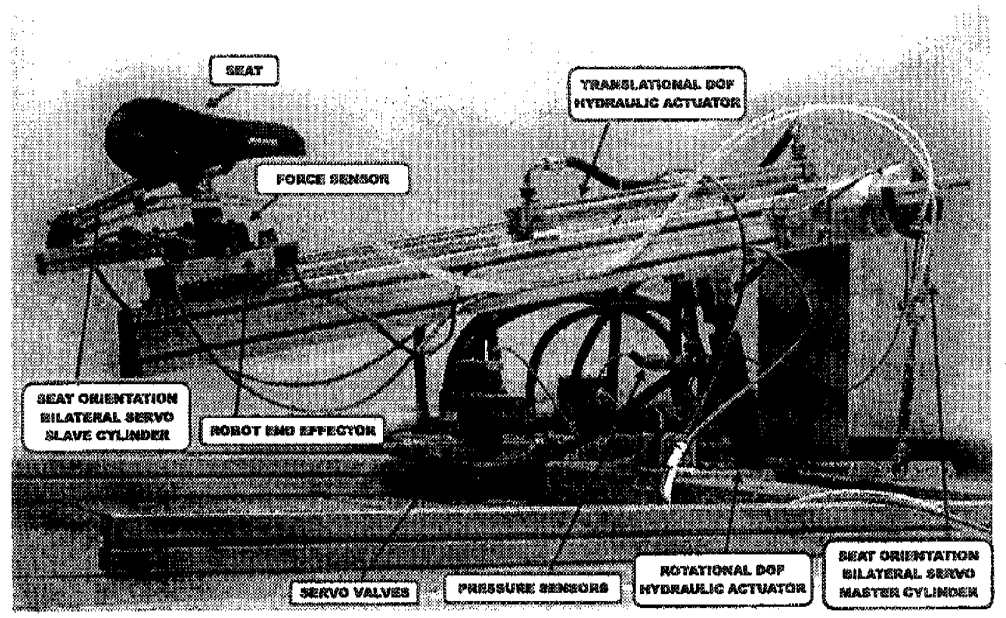

Figure 1: Standing up robot supportive device 
In the paper, a simulation model of the robot supported standing-up of paraplegic subject is presented. The behaviour of two systems that interact with each other is modeled. The body of parapelgic person, as the first system, is presented by a closed kinematic body chain including upper body voluntary controlled joints and the lower body joints that are passive. The robot, as the second system, is modeled as a mechanical device accomplishing the desired motion trajectory regardless the interaction between the subject and the robot. The evaluation of the model was based on measurements of real robot supported rising of paraplegic subject. The model was designed for the analysis and comparison of different standing-up strategies.

\section{Methods}

\subsection{Measurements of Robot Supported Standing-Up}

The simulation model was developed on the basis of measurement data from the real robot supported standing-up. In the experiments, a person with paraplegia was involved (subject MT, female, 30 years, $171 \mathrm{~cm}, 74 \mathrm{~kg}$, injury level T 4-5). The subject was asked to accomplish several standing-up trials to demonstrate the interaction between the robot, the voluntarily controlled upper extremities, and paralyzed lower extremities. The start of the standing-up movement i.e. triggering of the robot was left to the subject. Triggering was realized via a pushbutton mounted on the walker handle. After triggering the robot accomplished the motion along a preprogramed end-point trajectory. The objective of the study was the assessment of the kinetic and kinematic parameters of the supported standing-up maneuver.

The kinematics of the body segments movement was measured by the OPTOTRAK optical system (Northern Digital Inc., Waterloo, Canada). The system measured the active markers (infrared LEDs) 3D positions attached to the human body anatomical landmarks. In Figure 2 the measurement setup is shown including the robot assistive device and the arm supportive frame.

External forces acting on the human body were assessed by the AMTI force plate (AMTI Inc., Massachusetts, USA), determining the ground reaction force vector, while a JR3 40E15 force sensor, mounted underneath the walker handle, was utilized for measuring the arm supporting forces. An additional JR3 force sensor, mounted underneath the robot seat, assessed the robot supportive forces. A three-dimensional inverse dynamic Newton-Euler formulation was used to compute the net joint moments and reaction forces from the acquired data. 


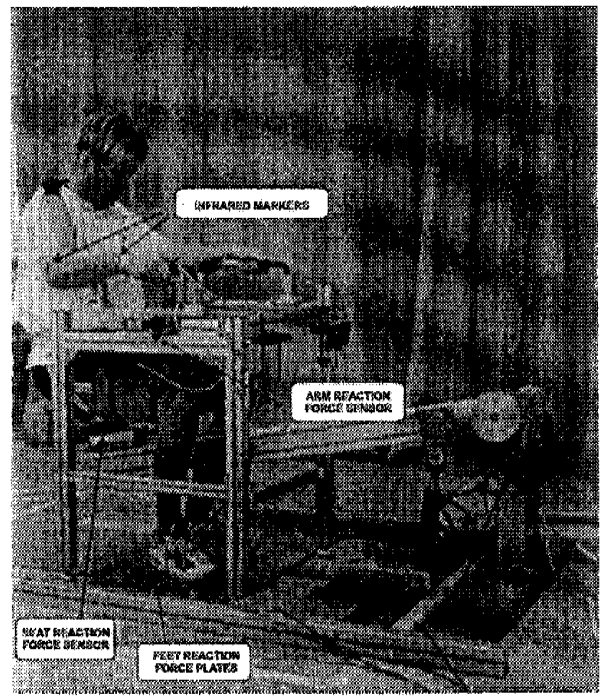

Figure 2: Person with paraplegia in the standing-up measurement setup

\subsection{Model of the Robot Supported Standing-Up}

In the simulation model, the human body is described with a two-dimensional model moving in the sagittal plane. The model consists of three rigid body segments embodying shanks, thighs and joined segments of head, arms and trunk HAT segment. The segments are interconnected by rotational pin joints. For each rigid body segment the length, mass, center of mass and inertia matrix are given. The trajectories of approximate joint centers were measured in real robot supported standing-up. Segment lengths are set to the mean value of the distance between two centers of the adjacent joints. The segment center of the mass is located on the straight line interconnecting two centers of the adjacent joints. The segment center of the mass is located on the straight line interconnecting two adjacent joint centers. The distance from the proximal joint to the center of the mass, the masses and the inertia matrices are determined according to the proportional anthropometric model in [9].

When modeling the motion of rigid bodies, all the forces and moments acting on the kinematic chain must be known. In rising, the HAT segment is supported by the lower and upper extremities in the hip and shoulder joints. Besides, robot interaction force is acting near the hip joints, while passive visco-elastic moments are acting in each body joint. The forces and moments acting to the HAT segment 

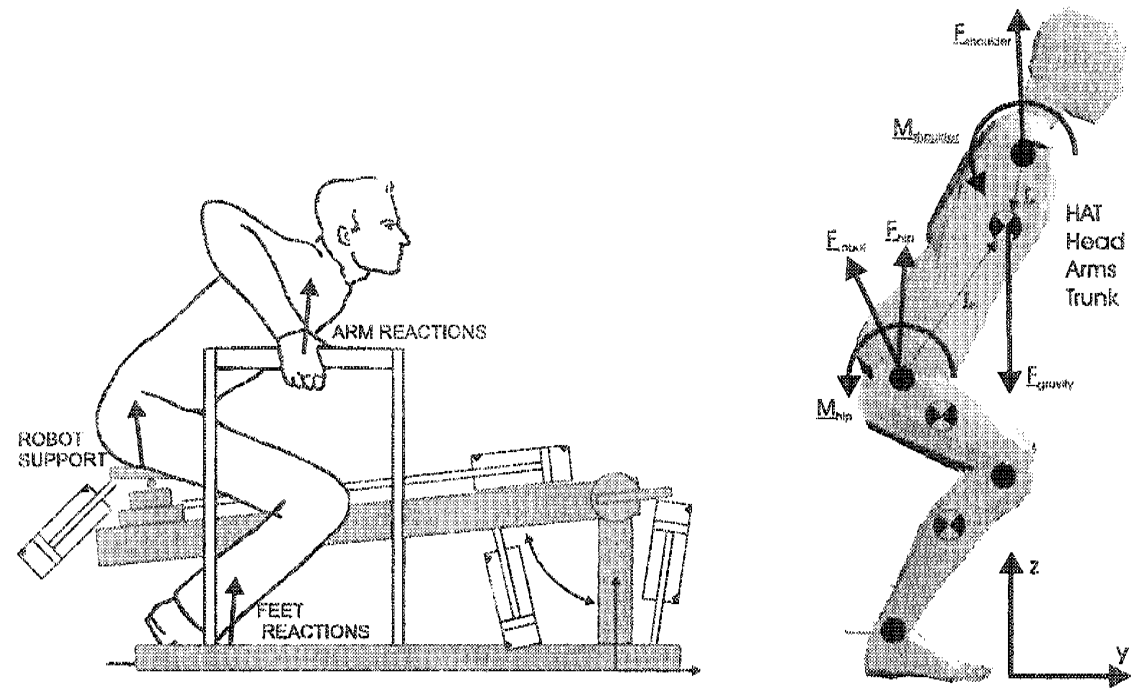

Figure 3: Supportive forces in robot supported standing-up

are illustrated in Figure 3 - right. As the paraplegic subject is not able to voluntary control the muscles of his lower extremities, only passive joint moments act around these joints. A passive joint moment is caused by deformation of the tissues surrounding the joint, such as relaxed muscles, ligaments, tendons, skin, etc.. Passive moments are often modeled with a nonlinear spring and a linear damper. Edrich with coauthors [11] proposed a comprehensive model of passive joint moments taking into account the angles of the adjacent, proximal $\left(\varphi_{\text {proxi }}\right)$ and distal $\left(\varphi_{\text {disti }}\right)$ joint:

$$
\begin{aligned}
M_{e i}= & e^{\left(k_{1 i}+k_{2 i} \varphi_{i}+k_{3 i} \varphi_{\text {proxi }}+k_{4 i} \varphi_{d i s t i}\right)} \\
& -e^{\left(k_{5 i}+k_{6 i} \varphi_{i}+k_{7 i} \varphi_{\text {proxi }}+k_{8 i} \varphi_{d i s t i}\right)} \\
& +e^{\left(k_{9 i} \varphi_{i}+k_{10 i}\right)}+k_{11 i}
\end{aligned}
$$

The exponential function $e^{\left(k_{9 i} \varphi_{i}+k_{10 i}\right)}$ is present only in the knee joint passive moment equation. This function models the effect of ligaments preventing the joint hyperextension. The constants $k_{i j}$ were in the study [11] derived from the measurement of a healthy subject.

The damping component around the joint is described by the equation:

$$
M_{d i}=-k_{12 i} \dot{\varphi}
$$


The total passive joint moment is then:

$$
M_{i}=M_{e i}+M_{d i}
$$

Paraplegic subjects make use of their arms during rising in order to achieve balance and to compensate for lag of lifting forces of the lower extremities. In this respect, Donaldson [10] showed that the trunk orientation in rising is fully controllable only by the upper extremities even in completely paralyzed patients who are not able to develop any support from the lower extremities. The arm support is modeled as a force and moment vector acting at the shoulder joint. Three degrees of freedom are thus under the influence of a voluntary effort: horizontal and vertical trunk position, and trunk orientation in the sagittal plane. For generating the shoulder forces and moment three independent PD controllers were implemented (see Figure 4). PD controllers applied the vertical and horizontal shoulder force, and the moment in the sagittal plane according to the displacement between the desired and actual trunk position and orientation. Because it was assumed that the patient tries to follow a trajectory he had learned, the desired trunk trajectory was obtained from the real experimental data.

The standing-up robot was modeled as a stiff device performing the seat positioning in the sagittal plane. The seat is positioned according to the reference position and velocity profiles. The reference trajectory was extrapolated from the measurement data. When operating in a position control mode, no interaction control is supposed to be incorporated in the robot controller.

Exponential springs and linear dampers were used to model the dynamic interaction between the human model and the robot seat [12]. The hip joint was constrained to move in the forward and backward directions by one spring and damper, and in the downward direction by a second spring and damper. The details for computing the horizontal and vertical components of robot supportive force exerted on the human body model are given below:

$$
\begin{gathered}
f_{\text {robot }, y}= \begin{cases}0.5\left(10^{(450|\Delta y|)}\right)-3000 \dot{y} & \Delta y \leq 0 \\
-0.5\left(10^{(450|\Delta y|)}\right)-3000 \dot{y} & \Delta y>0\end{cases} \\
f_{\text {robot }, z}= \begin{cases}0.5\left(10^{(450|\Delta z|)}\right)-3000 \dot{z} & \Delta z \leq 0 \\
0.0 & \Delta z>0\end{cases}
\end{gathered}
$$

where:

$\Delta y=y_{\text {hip }}-y_{\text {seat }}$ is horizontal displacement between hip and seat, $\Delta z=z_{\text {hip }}-z_{\text {seat }}$ is vertical displacement between hip and seat, $\dot{y}$ is horizontal velocity of hip, $\dot{z}$ is vertical velocity of hip. 


\subsection{Simulation Environment}

Deriving the equations of motion of a complex system in analytic form takes considerable time. For this reason the SD/FAST (Symbolic Dynamics, Inc., USA) software packages was used. The SD/FAST allows a dynamic analysis of mechanical systems which are represented as a chain of rigid body segments interconnected by joints. Inverse dynamics, direct dynamics or combination of both problems can be computed. The SD/FAST derives the software functions which are utilized in computation of motion derivatives and outputs. The development of functions is based on the Kane's formulation [13]. The equations of motion must be completed with proper forces and moments acting on the kinematic chain. A program in programing language $\mathrm{C}$ was therefore written incorporating forces and moments applied in each integration step. In this way, the passive joint moments, robot interaction force and arm force were applied in the model.

The Matlab-Simulink software package (The Math Works, Inc., Natic, MA) was used to simulate the robot supported sit-to-stand process. The Simulink environment served for integration of the system states, display and animation of the results. Into the Simulink, a dynamic model of the human body was integrated via $\mathrm{S}$-function interface. In Figure 4 a block scheme of the simulation concept is presented.

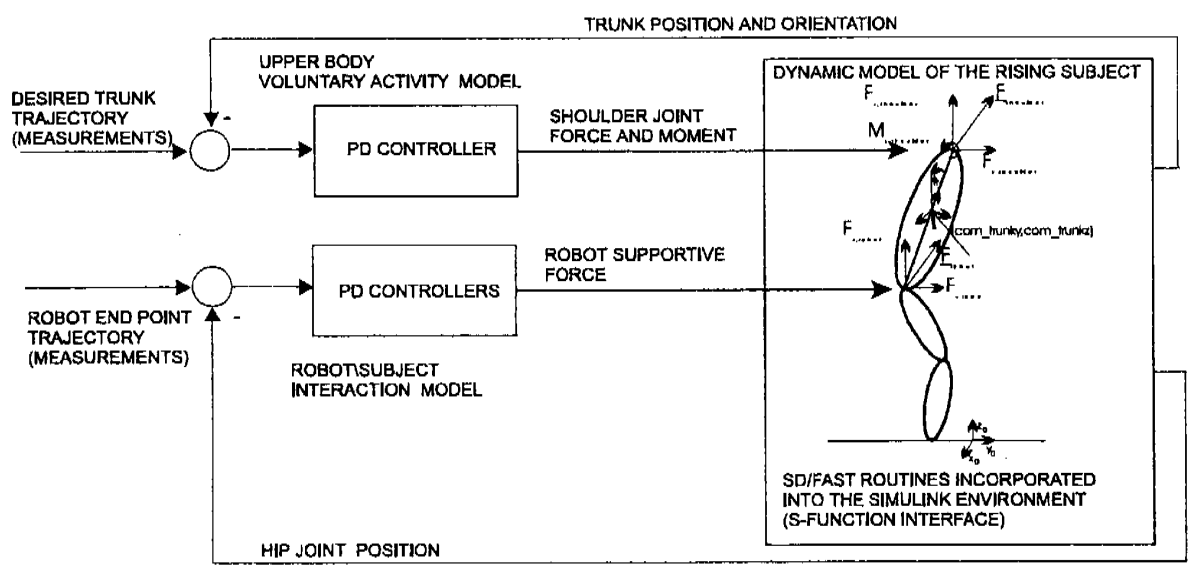

Figure 4: Block scheme of simulation concept 


\section{Evaluation of the Simulation Model}

The simulation model was evaluated by the help of experimental data acquired in real robot supported standing-up of paraplegic subject. The model responses were compared to the results of 3-dimensional inverse dynamic study. As a reference, the measured trunk trajectory and robot seat trajectory were fed to the simulator. The human body motion, shoulder joint forces and moment, and the robot interaction force were calculated as model outputs.

The vertical arm force acting at the shoulder joint and the vertical robot reaction force acting at the hip joint are presented bellow in Figure 5. Seat reaction force demonstrates the amount of robot support, while the arm force reflects the subject's voluntary activity assuring body balance and providing lifting forces. In Figure
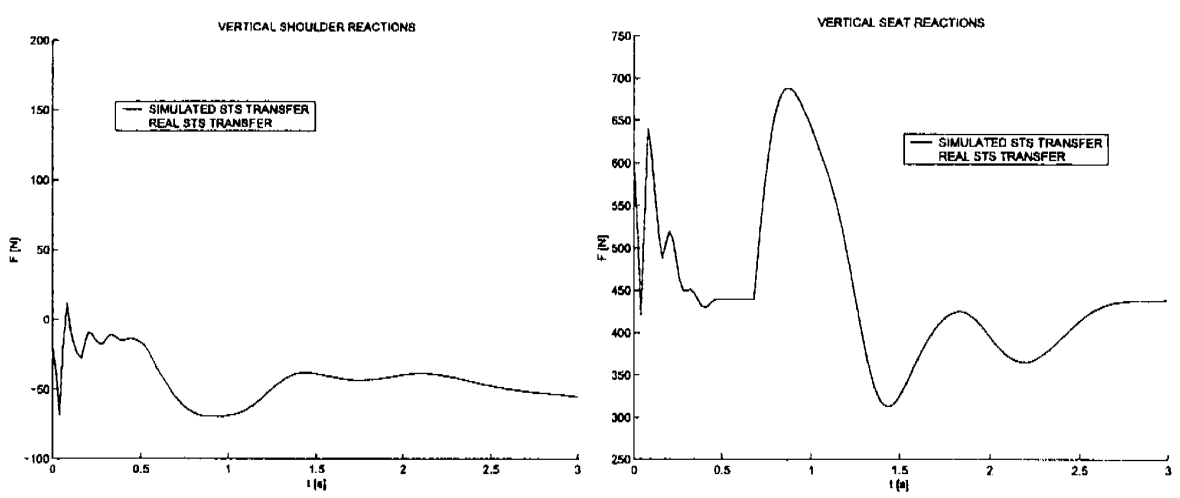

Figure 5: Voluntary activity (shoulder joint loadings) and robot support (seat reactions) in simulated and real robot supported standing-up of paraplegic subject

6 the body positions are shown in particular time instants of a single standingup trial. For each time instant, the body positions are compared acquired from a simulated and real robot supported standing-up. They are presented with the black and white figures, respectively.

\section{Discussion and Conclusions}

The simulation model of robot supported standing-up of paraplegic person was developed and implemented in the Matlab-Simulink simulation environment. The inputs to the model are the desired trajectory of the upper body and the robot seat 


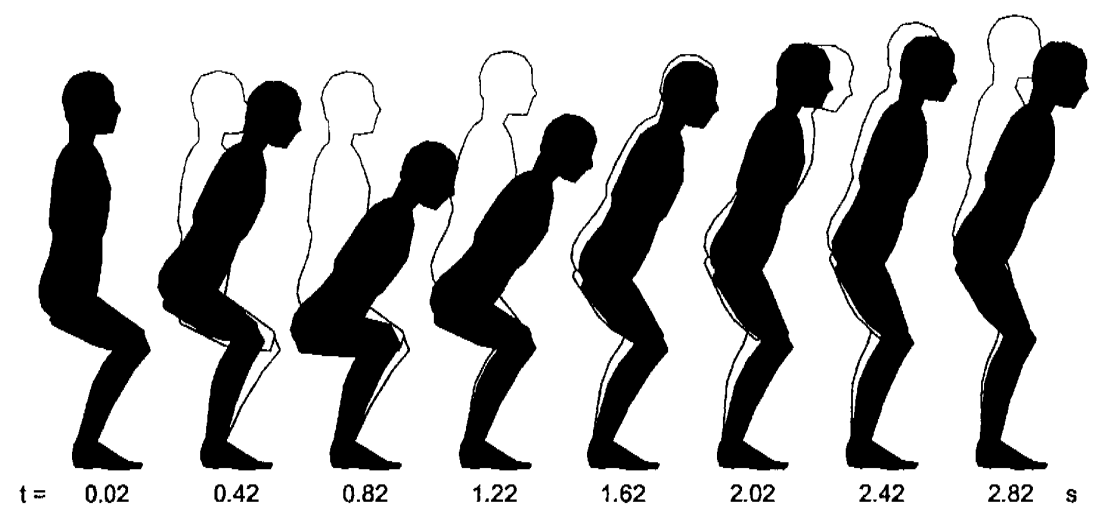

Figure 6: Measured and simulated body movement during robot supported rising from a sitting to a standing position

trajectory. In the model, the arm and robot supportive forces are determined according to the human body dynamics. In this way the subject's volition in rising when interacting with the stiff robot device is modeled. The simulation model was evaluated by the measurement data of a real robot supported standing-up of paraplegic subject. The evaluation results showed that the model behaves reliably. In the experimental standing-up the effect of taking over the support by the arms after attaining the standing posture is evident. This effect is attributed to the subject's intention to balance the standing posture and is therefore not incorporated in the standing-up simulation model.

The simulation model of the robot supported standing-up was designed to study different standing-up strategies. Different subject's approach to the rising maneuver can simply be obtained by varying the desired trunk trajectory at the simulator input. Moreover, additional aids which are being used to facilitate the standing up of paraplegic subjects (e.g. functional electrical stimulation -FES) can also be evaluated by means of simulation. Another possible application of the simulation model is to verify different control approaches of the robot controller, as it is proposed in [14], for example. Therefore, as an extension of the presented work, we propose incorporation of the dynamics of robot mechanical structure and hydraulic actuation system into the simulation model. 


\section{References}

[1] Schultz, A.B., Alexander, N.B. \& Ashton-Miller, J.A. Biomechanical analyses of rising from a chair. J. Biomech., vol. 25, pp. 1383-1391, 1992.

[2] Ellis, M.I., Seedhom, B.B. \& Wright, V. Forces in the knee joint whilst rising from a seated position. J. Biomed. Eng., vol. 6, pp. 113-128, 1984.

[3] Bahrami, F., Riener, R., Jabedar-Maralani, P., \& Schmidt, G. Biomechanical analysis of sit-to-stand transfer in healthy and paraplegic subjects. Clinical Biomechanics, Vol. 15, No. 2, pp. 123-133, 2000.

[4] Gellman, H., Sie, I. \& Waters, R.L. Late complications of the weight bearing upper extremity in the paraplegic patient. Clin. Orthop. Relat. Res., vol. 233, pp. 132-135, 1998.

[5] Munro, B.J., Steele, J.R., Bashford, G.M., Ryan, M. \& Britten, N. A kinematic and kinetic analysis of the sit-to-stand transfer using an ejector chair: implications for elderly rheumatoid arthritic patients. J. Biomech, vol. 31, pp. 263-271, 1998.

[6] Wretenberg, P., Arborelius, U.P., Weidenhlelm, L., \& Lindberg, F. Rising from a chair by a spring-loaded flap seat: a biomechanical analysis, Scand. J. Rehabil. Med., vol. 25, pp. 153-159, 1993.

[7] Schüldt, K., Ekholm, J., Németh, G., Arborelius, U.P. \& Harms-Ringdahl, K. Knee load and muscle activity during exercises in rising. Scand. J. Rehabil. Med., vol. 15, suppl no, 9, pp. 174-199, 1983.

[8] Riener, R., Ferrarin, M., Pavan, E.E., \& Frigo, C.A. Patient-driven control of FESsupported standing up and sitting down: experimental results. IEEE Trans. Rehabil. Eng., vol. 8, pp. 523-529, 2000.

[9] De Leva, P. Adjustments to Zatsiorksy-Seluyanov's segment inertia parameters, $J$. Biomech., vol. 29, pp. 1223-1230, 1996.

[10] Donaldson, N. de N., \& Ya, C.-H. FES standing: control by handle reactions of leg muscle stimulation (CHRELMS). IEEE Trans. Rehabil. Eng., vol. 4, pp. 280-284, 1996.

[11] Edrich, T., Riener, R., \& Quintem, J. Analysis of passive elastic joint moments in paraplegics. IEEE Trans. Bio-Med. Eng., vol. 47, no. 8, pp. 1058-1065, 2000.

[12] Pandy, M.G., Gamer, B.A., \& Anderson, F.C. Optimal control of non-ballistic muscolar movements: A constraint-based performance criterion for rising from a chair. $J$. Biomech. Eng., vol. 117, pp. 15-26, 1995.

[13] Kane, T.R., \& Levinson, D.A. Dynamics: Therory and Applications. McGraw-Hill, Inc., New York, 1985.

[14] Kamnik, R., \& Bajd, T. Human voluntary activity integration into the control of standing-up rehabilitation robot. Proc. of the 7th International Workshop on Advanced Motion Control, eds. pp. 402-407, IEEE: Maribor, Slovenia, Piscataway, 2002. 